\title{
Occurrence and prevalence of pulmonary tuberculosis in rural communities in Narsingdi, Bangladesh
}

\author{
Hafsa Afroz ${ }^{1}$, Md. Azhar Ali ${ }^{1,2}$, Selina Akter ${ }^{1}$, Zahed U.M. Khan ${ }^{1}$, and \\ Suvamoy Datta ${ }^{1}$ \\ ${ }^{1}$ Department of Microbiology, Primeasia University, Bangladesh \\ ${ }^{2}$ Upazilla Health Complex, Palash, Narsingdi, Bangladesh
}

\begin{abstract}
Tuberculosis is a chronic communicable disease. Infections are initially asymptomatic and latent but eventually progresses to active disease, which, if left untreated, may have $\geq 50 \%$ mortality. In 2011 , an antituberculosis drug resistance survey in Narsingdi, Bangladesh enrolled both new and retreatment patients. In the study, a number of villages were randomly selected and 264 pulmonary tuberculosis (PTB) patients were identified. All the patients received treatment with standardized first-line drug (FLD) regimens and were investigated to document treatment efficiency. Among the patients, 130 were reported in lower income, 117 in medium income and only 17 in higher income group. Farmers (95\%) in low income group, and female housewives (68\%) in medium income group were mostly infected. Silicosis and malnutrition were assumed responsible for high PTB cases in farmers and housewives respectively. Males were more prone to PTB and the ratios of male to female patients were found to increase with increasing age. The working youth group (25-34) and older people (above 64) were found most vulnerable. PTB patients in Bangladesh had high recurrence and death rates even after treatment with standardized FLD regimens, reinforcing the need for early survey, diagnosis and treatment, including assessment of treatment outcomes.
\end{abstract}

Keywords - Age, Bangladesh, Pulmonary tuberculosis, Socio-economic condition, Treatment efficiency

\section{Introduction}

Tuberculosis is a common and in many cases lethal infectious disease caused by various strains of Mycobacterium spp., usually Mycobacterium tuberculosis [1]. Most infections in humans result in an asymptomatic, latent infection and about one in ten latent infections eventually progresses to active disease. The mortality rate for untreated tuberculosis is more than $50 \%$. One third of the world's population is thought to have infected [2,3] and new infections occur at a rate of about one of two [2]. In 2007, an estimated 13.7 million chronic active cases, 9.3 million new cases, and 1.8 million deaths were reported and mostly in developing countries [4]. With over 9 million cases and nearly 2 million deaths annually, tuberculosis (TB) remains a major cause of morbidity and mortality worldwide. ${ }^{[6]}$ One of the important challenges for $\mathrm{TB}$ control is drug resistance, particularly multidrug resistant $\mathrm{TB}^{\left[{ }^{[7][8]}\right.}$ People with silicosis [5], chronic renal failure [6] and diabetes mellitus have a risk for developing active TB. People with diabetes mellitus are also at increased risk of contracting tuberculosis [7], and have suffered from poorer response to treatment [8]. Low body weight is associated with risk of tuberculosis [9]. Tuberculosis is the world's greatest infectious killer of women of reproductive age [10]. The incidence of TB varies with age as well. In Africa, TB primarily affects both adolescents and young adults [11]. However, in developed country of lower TB incidence, TB is mainly a disease of either older or immunocompromised people $[1,12]$. Diet also reported to modulate risk. Severe malnutrition in parts of the developing world causes a large increase in the risk of developing active tuberculosis immune system $[13,14]$. Along with overcrowding, poor nutrition may contribute to the strong link observed between tuberculosis and poverty $[15,16]$.

Diagnosis relies on radiology, tuberculin test, blood tests, as well as microscopic examination and microbiological culture of infected bodily fluids. Treatment is difficult and requires long courses of multiple antibiotics. Multi-drug-resistant tuberculosis threatens the treatment regimen further. Treatment for TB consist multiple antibiotic regimen. Effective TB treatment is difficult, due to the unusual structure and chemical composition of the mycobacterial cell wall, which makes many antibiotics ineffective and hinders the entry of drugs [17-20]. TB requires much longer periods of treatment (around 6 to 24 months) to entirely eliminate mycobacteria from the body [21] instead of the short course of antibiotics typically used. Latent TB treatment usually uses a single antibiotic, while active TB disease is best treated with combinations of several antibiotics, to reduce the risk of the bacteria developing antibiotic resistance [22]. Drug-resistant TB is a public health issue in many developing countries, as treatment is longer and requires more expensive drugs. Multi-drug-resistant tuberculosis (MDR-TB) is defined as resistance to the two most effective first-line TB drugs: rifampicin and isoniazid [23]. 


\subsection{Ethics Statement}

\section{Materials and Methods}

The study was approved by the Upazilla Health Complex, Palash, Narsingdi Ethical Committee for TB Operational Research. Written informed consent was obtained from individuals before an interview was conducted and sputum samples were collected. If the individual had died, written informed consent was obtained from the family member before they were interviewed. Patients and TB hospitals were informed of the sputum smear, culture, and DST test result. MDR-TB patients identified during follow-up were requested to go to the TB hospital for treatment, including with second-line drugs.

\subsection{Drug Resistance Survey}

For the drug resistance survey (DRS), the "Guidelines for surveillance of drug resistance in tuberculosis" developed by WHO/IUATLD were followed [24]. In the district Narsingdi, a number of villages were randomly selected under Palash Union council for the survey. All smear-positive cases in the survey area during the study period (From February to November, 2011) were diagnosed and positive samples were eligible for inclusion [25].

\subsection{Treatment Regimen and Outcome assessment}

The district Narsingdi followed the National Tuberculosis Programme (NTP) treatment guidelines for all TB patients. Treatment of new smear-positive patients consisted of 2 months of isoniazid, rifampicin, pyrazinamide, and ethambutol followed by 4 months of isoniazid and rifampicin three times weekly $\left(2 \mathrm{H}_{3} \mathrm{R}_{3} \mathrm{Z}_{3} \mathrm{E}_{3} / 4 \mathrm{H}_{3} \mathrm{R}_{3}\right)$. Patients who had previously received at least one month of TB treatment (i.e. retreatment patients) received 2 months of isoniazid, rifampicin, pyrazinamide, streptomycin and ethambutol, followed by 6 months of isoniazid, rifampicin, and ethambutol three times weekly $\left(2 \mathrm{H}_{3} \mathrm{R}_{3} \mathrm{Z}_{3} \mathrm{E}_{3} \mathrm{~S}_{3} / 6 \mathrm{H}_{3} \mathrm{R}_{3} \mathrm{E}_{3}\right.$.). Drug intake was observed at the health facility throughout the treatment.

Treatment outcomes were assessed using international definitions and were recorded in routine treatment registers [26]. Treatment success was defined as either cure or treatment completed. Cure was defined as a patient who was sputum smear-negative in the last month of treatment and on at least one previous occasion. Treatment completed was defined as a patient who had completed treatment but who did not meet the criteria to be classified as a cure or a failure. Treatment failure was defined as a patient who was sputum smearpositive at 5 months or later during treatment. Default was defined as a patient whose treatment had been interrupted for two consecutive months or more. Transfer out was defined as a patient who had been transferred to another recording and reporting unit and hence treatment outcome was unknown. Death was defined as a patient who died for any reason during the course of treatment. The cause of death during TB treatment was not recorded.

\subsection{Follow up of TB Patients}

Staffs from regional TB centers included in the DRS were instructed on how to follow-up the 264 patients included in the DRS. Regional staffs and one provincial staff member interviewed patients in their home in the period. During the interview, participants were asked about presence of common TB symptoms (i.e., cough, haemoptysis, chest pain, fever, fatigue, anorexia and night sweat). Three sputum samples were collected from all participants for microscopy, culture and DST using the same methods. Staff rechecked the smear examination results, diagnosis and dates with the medical files in the facility. If the patient had died, family members were interviewed about the time of death and its probable cause. Staff would recheck the cause with the death certificate provided by the hospital. Patients who had TB registered as a cause of death were assumed to have had recurrent TB.

\subsection{Data Management and Analysis}

Analysis was performed using SPSS 13.0 (SPSS Inc. Chicago, IL, USA). For the analysis of treatment outcome from initial TB treatment, we compared patients who were cured to those that failed, died, or defaulted in a multivariate logistic regression model. Variables available were living area, economic condition, sex, age, drug resistance pattern, bacillary load, and number of treatment episodes.

\section{Results and Discussion}

A total of $264 \mathrm{~TB}$ patients were recorded within the population of randomly selected villages under Palash Union council under Narsingdi district of Bangladesh. The survey regions were developing rural and sub-urban areas. Among the 264 TB patients, 130 patients were from low income group mostly of farmer (124 patients) along with a few patients from other occupation like day labour, rickshaw puller, bus driver and tailor (Figure 1). Medium income group population was also reported to have higher TB cases. A total of 117 patients were recorded in this economic group and most of them were of female sex and were housewives (68\%). 
Tuberculosis has already been considered as the world's greatest infectious killer of women of reproductive age [10]. The rest of the patients of this income group were either service holder (26\%) or students (10\%). TB cases from high income group were least reported and all those reported were business people.

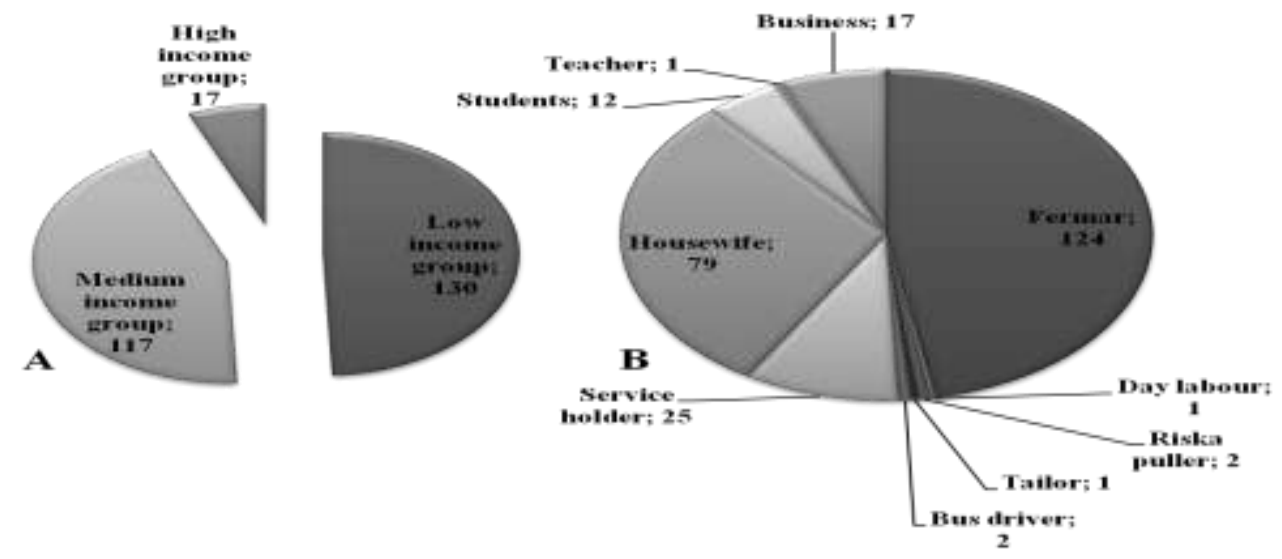

Figure 1: Graphical representation for the distribution of TB patients among different income groups residing in a number of randomely selected villeges in Narsingdi district, Bangladesh. A total of 264 patients were reported in the study region, among them 130 were reported in lower income group, 117 were in medium income group and only 17 patients were in higher income group (A). Occupation of the patients were also plotted (B) in each of the income groups.

The higher incidence of PTB in farmers can be due to the presence of silicosis in lungs in this occupational group. Silica particles, acquired during farming, irritate the respiratory system, causing immunogenic responses such as phagocytosis, which results in high lymphatic vessel deposits [27] and probably blockage of macrophage function. The phenomenon thus increases the risk of tuberculosis [28]. People with silicosis have an approximately 30 -fold greater risk for developing active TB [5].

The vulnerability to tuberculosis was found to vary between age and sex groups (Fig. 2 and Table 1). Males were found more prone to the disease than female and the incidence of infection increased in male in older age (Fig. 2). In the younger population (aged below 24), patient's sex were not a variable in their disease vulnerability. Males were reported to have higher TB incidence than female of aged above 25 . In the survey, there were a few cases of TB reported in children. The most vulnerable age groups were the youth working age group between aged 25 and 34 years along with the oldster population (Fig. 2). Similar observations have been reported earlier. In Africa, TB primarily affects adolescents and young adults [11]. However, in countries where TB has gone from high to low incidence, such as the United States, TB is mainly a disease of older people, or of the immunocompromised $[1,12]$.

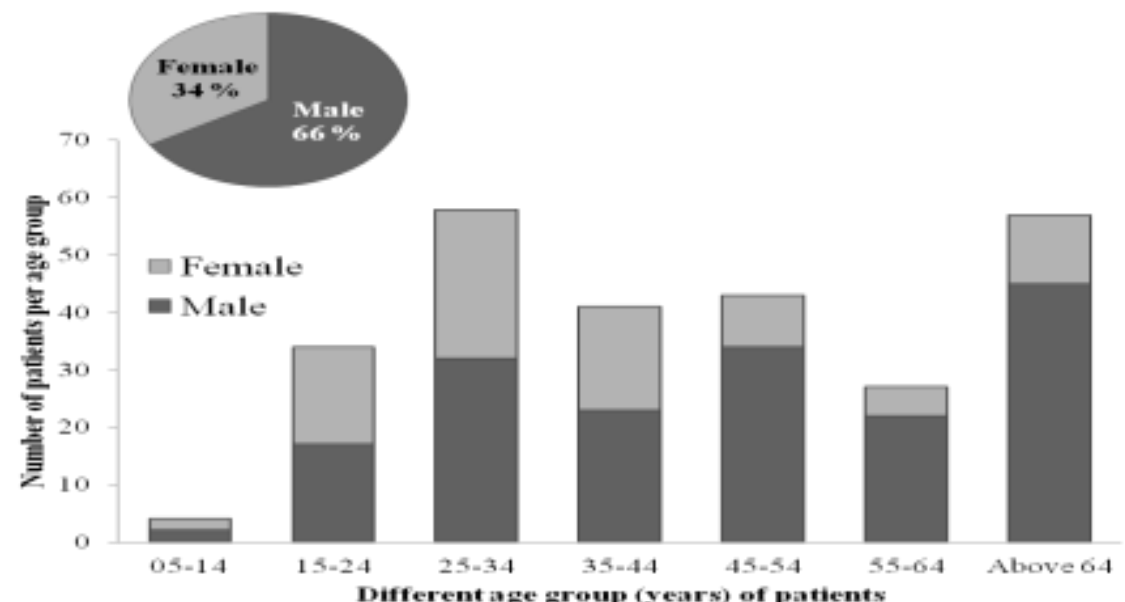

Figure 2: Graphical representation of TB patients in each of the age- and sex-groups. Among the 264 patients, males are dominating sex group (pie-diagram) reported to have TB in the study. The ratios of male to female patients were found to increase with the age of patients. The working youth group (25-34) and older people (above 64) were found more vulnerable to tuberculosis.

TABLE 1: The Follow-up of TB-patients Cured after 6-months Drug Regimen. 


\begin{tabular}{|c|c|c|c|c|c|c|c|c|c|c|}
\hline \multicolumn{2}{|c|}{$\begin{array}{c}\text { Age Group (years) of } \\
\text { patients }\end{array}$} & \multirow{2}{*}{ ஓ } & \multirow{2}{*}{$\frac{ \pm}{i n}$} & \multirow{2}{*}{$\begin{array}{l}\text { 志 } \\
\text { in } \\
16\end{array}$} & \multirow{2}{*}{$\frac{\text { ले }}{\text { in }}$} & \multirow{2}{*}{$\begin{array}{l}\text { \& } \\
\text { ñ } \\
22\end{array}$} & \multirow{2}{*}{ 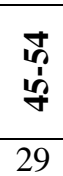 } & \multirow{2}{*}{$\begin{array}{l}\text { J্ } \\
1 \\
1 \\
\text { ñ } \\
17\end{array}$} & \multirow{2}{*}{$\frac{8}{8}$} & \multirow{2}{*}{$\frac{\stackrel{5}{0}}{150}$} \\
\hline Patients cured & Male & & & & & & & & & \\
\hline after 2 months of & Female & - & - & 13 & 24 & 16 & 9 & 5 & 10 & 77 \\
\hline drug regimen & Sum up & - & - & 29 & 49 & 38 & 38 & 22 & 51 & 227 \\
\hline \multirow{3}{*}{$\begin{array}{l}\text { Patients cured } \\
\text { after } 5 \text { months of } \\
\text { drug regimen }\end{array}$} & Male & - & - & - & 3 & 1 & 5 & 3 & 3 & 15 \\
\hline & Female & - & - & - & - & - & - & - & 2 & 2 \\
\hline & Sum up & - & - & - & 3 & 1 & 5 & 3 & 5 & 17 \\
\hline \multirow{3}{*}{$\begin{array}{l}\text { Patients cured } \\
\text { after } 6 \text { months of } \\
\text { drug regimen }\end{array}$} & Male & - & 2 & 1 & 4 & - & - & 2 & 1 & 10 \\
\hline & Female & - & 2 & 4 & 2 & 2 & - & - & - & 10 \\
\hline & Sum up & - & 4 & 5 & 6 & 2 & - & 2 & 1 & 20 \\
\hline \multirow{3}{*}{$\begin{array}{l}\text { Patients not } \\
\text { cured/treatment } \\
\text { failed/default/died }\end{array}$} & Male & - & - & - & - & - & - & - & - & - \\
\hline & Female & - & - & - & - & - & - & - & - & - \\
\hline & Sum up & - & - & - & - & - & - & - & - & - \\
\hline Total & & - & 4 & 34 & 58 & 41 & 43 & 27 & 57 & 264 \\
\hline
\end{tabular}

In the survey, patients diagnosed to be TB positive were treated and followd up. All the patients were first administered with 2 months drug regime, for new cases the drug regime consisted of isoniazid, rifampicin, pyrazinamide, and ethambutol and for the patients who received at least one month TB treatment previously the drug regime consisted of isoniazid, rifampicin, pyrazinamide, streptomycin and ethambutol. Upon the regime, $86 \%$ patients were responded to the treatment and diagnosed to be cured. The treatment were completed with another drug regime; 4 months drug consisted of isoniazid and rifampicin for newly diagnosed patients and 6 months drug consisted of isoniazid, rifampicin, and ethambutol for previously treated patients. Drug intake and responses to drugs were observed at the health facility throughout the treatment. Within 6 months from starting treatment, all the 264 patients were reported to be cured. No cases of treatment failure, treatment default or death was reported among the TB patients in the study period.

\section{Conclusion}

In Bangladesh, 300000 new cases and 70000 deaths reported per year due to PTB [29], hence the nationwide survey on its prevalence got importance in recent years. There was an anti-tuberculosis drug resistance survey performed in the district of Narsingdi in Bangladesh in 2011. Both the new and retreatment patients were included in the survey. The study was based on the information collected from 264 pulmonary tuberculosis (PTB) patients, which were identified in a number of randomly selected villages among the survey area. The patients were diagnosed, received treatment with FLD regimens and treatment efficiency were investigated by follow up procedure. Living standard and economic conditions was found to influence the prevalence. PTB had also found to infect variably with gender and in different age groups. Farmers were mostly suffered from PTB among the low income group and female housewives among the medium income group. Males were more vulnerable than female to PTB and it increases with ageing. The working youth and elderly people were found the most vulnerable. PTB patients in Bangladesh had already been reported to have high recurrence and death rates, which reinforced need for early survey, diagnosis, treatment and outcome assessment. Though, the study had given a scenario on the PTB prevalence in Bangladesh, regional and geographical impact could be assessed by long term countrywide investigation.

\section{Acknowledgements}

We are pleased to acknowledge the Thana Health Complex, Palash, Narshingdi, Bangladesh for the assistance in the survey and providing information under 'Anti-tuberculosis drug resistance survey'.

\section{References}

[1] V. Kumar, A.K. Abbas, N. Fausto, and R.N. Mitchell, Robbins Basic Pathology (8th ed.), (UK, Saunders Elsevier, 2007), 516-522.

[2] WHO, Tuberculosis fact sheet $N^{\circ} 104$, (World Health Organization, 2010).

[3] R.M. Jasmer, P. Nahid, and P.C. Hopewell, Clinical practice. Latent tuberculosis infection, N. Engl. J. Med., 347(23), 2002, 18601866.

[4] WHO, Global tuberculosis control: epidemiology, strategy, financing, in Epidemiology, (World Health Organization, 2009 ), 6-33.

[5] ATS/CDC Statement Committee on Latent Tuberculosis Infection, Targeted tuberculin testing and treatment of latent tuberculosis infection. American Thoracic Society, MMWR Recomm Rep., 49(RR-6), 2000, 1-51.

[6] L. Segall, and A. Covic, Diagnosis of tuberculosis in dialysis patients: current strategy, Clin J Am Soc Nephrol, 5(6), 2010, 11141122 .

[7] B.I. Restrepo, Convergence of the tuberculosis and diabetes epidemics: renewal of old acquaintances, Clin Infect Dis., 45(4), 2007, 436-438. 
[8] H.M.J. Nijland et al., Exposure to rifampicin is strongly reduced in patients with tuberculosis and type 2 diabetes, Clin Infect Dis., 43(7), 2006, 848-854.

[9] C.C. Leung, T.H. Lam, W.M. Chan et al., Lower risk of tuberculosis in obesity, Arch. Intern. Med. 167(12), 2007, $1297-1304$.

[10] Stop TB Partnership-2002, London tuberculosis rates now at Third World proportions, (PR Newswire, Europe. http://www.prnewswire.co.uk/cgi/news/release?id=95088, Retrieved 3 October 2006).

[11] WHO, Global Tuberculosis Control Report, 2006 (Annex 1), Profiles of high-burden countries, http://www.who.int/tb/publications/global_report/2006/pdf/full_report_correctedversion.pdf. Retrieved 13 October 2006).

[12] Centers for Disease Control and Prevention (CDC), (Surveillance Slide Set, http://www.cdc.gov/nchstp/tb/pubs/slidesets/surv/surv2005/default.htm. Retrieved 13 October 2006).

[13] U.E. Schaible, S.H. Kaufmann, Malnutrition and infection: complex mechanisms and global impacts, PLoS medicine, 4(5), 2007, e115.

[14] K. Lönnroth, and M. Raviglione, Global epidemiology of tuberculosis: prospects for control, Seminars in respiratory and critical care medicine, 29(5), 2008, 481-491.

[15] P.D. Davies, The world-wide increase in tuberculosis: how demographic changes, HIV infection and increasing numbers in poverty are increasing tuberculosis, Annals of medicine, 35(4), 2003, 235-243.

[16] D.P. Spence, J. Hotchkiss, C.S. Williams, and P.D. Davies, Tuberculosis and poverty". BMJ (Clinical research ed.), 307(6907), 1993, 759-761.

[17] N.P.V. Acharya, M. Senn, and E. Lederer, Sur la presence et structure de mycolate d'arabinose dans les lipides lies de deux souches de Mycobacteries, Compte Rendu Acad Sci Hebd Acad Sci D., 264, 1967, 2173-2176.

[18] D. Migliore, N.P.V. Acharya, and P. Jolles, Characterization of large quantities of glutamic acid in the walls of human virulent strains of mycobacteria, Compte Rendu Acad Sci Hebd Acad Sci D., 263(11), 1966, 846-848.

[19] N.P.V. Acharya, D.S. Goldman, Chemical composition of the cell wall of the H37Ra strain of Mycobacterium tuberculosis, J Bacteriol, 102(3), 1970, 733-739.

[20] P.J. Brennan, H. Nikaido, The envelope of mycobacteria, Annu. Rev. Biochem., 64, 1995, $29-63$.

[21] Centers for Disease Control and Prevention (CDC), Core Curriculum on Tuberculosis: What the Clinician Should Know, (CDC, Division of Tuberculosis Elimination, http://www.cdc.gov:80/tb/pubs/corecurr/default.htm., updated August 2003).

[22] R. O'Brien, Drug-resistant tuberculosis: etiology, management and prevention, Semin Respir Infect., 9(2), $1994,104-12$.

[23] Centers for Disease Control and Prevention (CDC), Emergence of Mycobacterium tuberculosis with extensive resistance to secondline drugs - worldwide, MMWR Morb Mortal Wkly Rep., 55(11), 2004, 301-305.

[24] WHO, Guidelines for surveillance of drug resistance in tuberculosis, (Geneva, Switzerland: World Health Organization, 1997)

[25] Y.G. Xie, F.B. Li, and X.L. Yan, WHO TB drug resistance survey in Heilongjiang province, The Journal of the Chinese Antituberculosis Association, 30, 2005, 395-398.

[26] WHO, Treatment of tuberculosis: guidelines for national programmes ( $3^{\text {rd }}$ Edition), (Geneva, Switzerland: World Health Organization, 2003).

[27] J.H. Lee, Tuberculosis and silicosis, Can Med Assoc J., 58(4), 1948, 349-353.

[28] Varkey B, Silicosis, http://emedicine.medscape.com/article/302027-overview\#a0199, Retrieved 26 July 2011.

[29] WHO, Tuberculosis and health sector reforms in Bangladesh, (New Delhi, India: World Health Organization, Regional office for South-East Asia, 2004). 